\title{
Imaging Technologies - A Tool for Early Breast Cancer Diagnostic
}

\author{
Arya Bhushan* \\ Ladue Horton Watkins High School, St. Louis, Missouri, USA
}

*Corresponding Author: Arya Bhushan, Ladue Horton Watkins High School, St. Louis, Missouri, USA

\begin{abstract}
Breast cancer is still the most common cancer worldwide after cardiovascular diseases. The incidence of breast cancer has been increasing by $3.1 \%$ every year and this trend is expected to continue. During their life times about $12 \%$ of women suffer from this disease. Although several aggressive treatment strategies used for breast cancer, the rate of mortality has remained high and thus, there is a need to develop new approaches for cancer management. Molecular Imaging is one of the major tools used in comprehensive breast cancer care. Researchers have studied many breast diagnostic approaches, including mammography, ultrasonography, magnetic resonance imaging, scintimammography, single photon emission computed tomography and positron emission tomography. Early-stage cancer detection could reduce breast cancer death rates significantly in the long-term. Identifying early-stage cancer cells is critical for an optimal prognosis. Technological advances in imaging are creating new opportunities for improvements in both screening and early detection. This review article aims to provide an overview on recent important achievements in breast screening methods for rapidly diagnosing and managing breast cancer.
\end{abstract}

Keywords: Breast Cancer (BC); Magnetic Resonance Imaging (MRI); Computed Tomography (CT); and Positron Emission Tomography (PET)

\section{INTRODUCTION}

Breast cancer is the most frequently diagnosed cancer and the most frequent cause for cancer-related deaths in women worldwide. Breast cancer will be diagnosed in $12 \%$ of women in the United States over the course of their lifetimes and more than 250,000 new cases of breast cancer were diagnosed each year since 2017 in the United States. In the last decade, the number of new cancer cases went up about $21 \%$ in women, which is about 900,000 cases per year worldwide. Out of all types of cancers, breast cancer is the most common cancer and the second most common cause of cancer death in women after lung cancer. According to the U.S. Breast cancer statistics, 1 in 8 women in America will be diagnosed with breast cancer in their lifetime at every 2 minutes and 1 woman will die of breast cancer every 13 minutes. ${ }^{1}$ In order to cure this disease from its roots, there is a need to diagnose breast cancer in its earliest stage. Imaging of the breast is utilized almost exclusively for detection, diagnosis, and clinical management of cancer and for the assessment of the integrity of breast implants. Commonly used imaging modalities include mammography, ultrasonography, magnetic resonance imaging (MRI), scintimammography, single photon emission computed tomography (SPECT), and positron emission tomography (PET). The goal of this review is to collect literature which provides brief information about breast cancer and a prospective view of molecular imaging in breast cancer, including MRI and Radiation Imaging, primarily for detection and diagnosis, in order to stop the cancer in its earliest stages.

\section{DISCUSSION}

\subsection{Molecular Imaging and Breast Cancer}

Molecular imaging is a type of medical imaging that provides detailed pictures of what is happening inside the body at the molecular and cellular level. Whereas other diagnostic imaging proceduressuch as x-rays, computed tomography (CT) and ultrasound - predominantly offer anatomical pictures, molecular imaging allows physicians to see how the body is functioning and to measure its chemical and biological processes. In terms of diagnosis, molecular imaging is able to provide information without invasive procedures such as a biopsy or surgery. It also helps identify disease in its earliest stages and determine the exact location of a tumor, often before symptoms occur or abnormalities can 
be detected with other diagnostic tests. This helps to determine the extent or severity of the disease, including whether it has spread elsewhere in the body. This information can be used to select the most effective therapy based on molecular properties of a tumor and determine a response for the patient, accurately assess the effectiveness of a treatment and adapt treatment plans quickly in response to changes in cellular activity, and evaluate progression to identify recurrence of disease and help manage ongoing care. Molecular imaging procedures are mainly noninvasive, safe and painless which involve an imaging device, an imaging agent, or probe. To visualize cellular activity, such as the chemical processes involved in metabolism, oxygen use or blood flow, a variety of imaging agents are used. In nuclear medicine, the imaging agent is a radiotracer, a compound that includes a very small amount of a radioactive atom, or isotope. Other molecular imaging modalities, such as optical imaging and molecular ultrasound, use a variety of different agents. MRI is able to measure chemical levels in the body, without the use of an imaging agent. Once the imaging agent is introduced into the body, it accumulates in a target organ and attaches to specific cells. The imaging device detects and creates pictures to show how the imaging agent is distributed in the body; this distribution pattern helps physicians discern how well organs and tissues are functioning. In this article we are currently focused on MRI and few Nuclear molecular imaging technologies used for breast cancer. ${ }^{2}$

\subsection{Magnetic Resonance Imaging (MRI)}

Breast Magnetic Resonance Imaging (MRI) uses radio waves and strong magnets to take detailed pictures inside of the breast which used to help measure the size of the cancer, look for other tumors in the breast, and to check for tumors in the opposite breast in women who already have been diagnosed with breast cancer. The use of MRI for breast was introduced 40 years ago ${ }^{3}$ where initial images were disappointing, but intravenous contrast ${ }^{4}$ and faster sequences have changed this over the period. MRI is now standard tool for screening women who are at high risk of breast cancer. Key to the developing use of MRI has been the development of MRI-guided biopsy techniques. ${ }^{5}$ In the mid1990 s with the goal of eliminating the sampling difficulties associated with core needle biopsy Vacuum-assisted biopsy (VAB) devices came onto the market which facilitates removal of microcalcifications and is associated with less frequent under diagnosis of malignancies. ${ }^{6} \mathrm{VAB}$ is currently recommended for stereotactic and MRI-guided interventions. ${ }^{7}$ Several indications for MRI of the breast, including screening the high-risk population, have been recommended. ${ }^{8}$ Because of the method's high sensitivity breast malignancies that usually escape clinical, ultrasound, and mammographic detection can be detected by MRI. Because of the high sensitivity and lower specificity of breast MRI, it is imperative that these findings be histologically assessed before any surgical intervention. ${ }^{9,10}$

Dynamic Contrast Enhanced MRI (DCE-MRI) is a non-invasive imaging technique is primarily based on lesion contrast-agent-enhancement velocity giving sequential images during the passage of a contrast agent within a tissue of interest. The rapid enhancement seen in carcinomas is thought to be due to the angiogenic potential of malignant lesions. Angiogenesis is a requirement of neoplastic growth, progression, and metastasis. For breast cancer, contrast-enhanced breast MRI is a good imaging modality to depict tumor angiogenesis because the contrast enhancement pattern of the tumor correlates with micro vessel density characteristic of tumor angiogenesis. In addition, DCE-MRI features such as peripheral rim enhancement, faster enhancement, and washout curve type are correlated with overall recurrence and overall survival of breast cancer patients. ${ }^{11}$ DCE-MRI is an evolving tool for determining breast disease, which benefits from the move to imaging at $3 \mathrm{~T}$. It has major capabilities for the diagnosis, detection and monitoring of malignancy. ${ }^{12}$ It benefits from being non-invasive and three-dimensional, allowing visualization of the extent of disease and its angiogenic properties, visualization of lesion heterogeneity, detection of changes in angiogenic properties before morphological alterations, and the potential to predict the overall response either before the start of therapy or early during treatment. ${ }^{13}$ It was shown that increased predictive value could be obtained when integration of both morphology and contrast uptake dynamics is taken into consideration. ${ }^{14}$

MR Elastography (MRE) is a novel non-invasive technique capable of quantifying tissue mechanical properties in vivo, used to measure the spatial stiffness of soft tissues. Noninvasive MRE is Elastography can be simply described by three steps: deforming the tissue of interest through application of mechanical waves, imaging the subsequent deformation, and calculating the corresponding mechanical properties. ${ }^{15}$ MRE is well suited for breast cancer diagnosis and staging as a means to quantify the properties currently assessed by manual palpation. Some studies have 
documented the differentiation between normal, malignant, and benign tumors. ${ }^{16}$ This may overcome the limitations of manual palpation and increase the DCE-MRI specificity as well. ${ }^{17}$ While the initial results are encouraging, the most significant limitation for MRE in breast cancer is spatial resolution and detection of small focal lesions which can be improved by applying with application of 3D imaging and inversion algorithms. Further studies needed to define the utility of this technique in differentiating benign and malignant breast pathology. Over the past decade, there has been increasing interest in the use of Diffusion Weighted Imaging (DWI) for oncology applications. DWI is an advanced MRI technique that can measure the mobility of water molecules diffusing in tissue, which is impacted by biophysical characteristics such as cell density, membrane integrity, and microstructure. ${ }^{18}$ DWI is increasingly being incorporated into breast MRI protocols to address some of the shortcomings of routine clinical breast MRI. Potential benefits include improved differentiation of benign and malignant breast lesions, assessment and prediction of therapeutic efficacy, and noncontrast detection of breast cancer. ${ }^{19}$ The breast presents a unique imaging environment with significant physiologic and inter-subject variations, as well as specific challenges to achieving reliable high-quality diffusion weighted MR images. ${ }^{20}$ Technical advantages of DWI include a short acquisition time (typically 2-3 minutes), wide availability on most commercial scanners, and no need for administration of any contrast agent. Given these advantages and the ability to measure distinct and possibly complementary biological properties to conventional DCE- MRI, DWI increasingly has been studied for breast imaging applications. Promising data from multiple single center studies demonstrate value of DWI for the detection and characterization of breast cancer. ${ }^{21}$ Technical innovations are helping to overcome many of the image quality issues that have limited widespread use of DWI for breast imaging. Advanced modeling approaches to further characterize tissue perfusion, complexity, and glandular organization may expand knowledge and yield improved diagnostic tools. ${ }^{22}$ DWI may be an accurate and nonradioactive imaging technique for identifying breast cancer. Nonetheless, there are a variety of issues when assessing DWI techniques for estimating breast cancer responses, and large scale and well-designed clinical trials are needed to assess the technique's diagnostic value.

A technique called in vivo Magnetic resonance spectroscopy (MRS) is an additional technique that can be used to improve the accuracy of a breast MRI study. Over the last two decades, in vivo proton (1H) MRS has been shown to have the potential as a non-invasive tool for diagnosis and to provide an insight into the biochemistry of living tissues. Most in-vivo MRS studies carried out and reported in the literature on breast used either proton $(1 \mathrm{H})$ or phosphorus $(31 \mathrm{P})$ nucleus owing to their high natural abundance and sensitivity. 1H MRS not only offers higher sensitivity compared to 31P, but also has the ability to provide biochemical information from a well-defined region of interest or voxel, using volume localization method. ${ }^{23}$ The addition of in-vivo 1H MRS protocol with the MRI procedure increases the overall acquisition time by approximately 10 minutes but has the advantage to improve the diagnostic accuracy of clinical breast MR, thus making it a more versatile and widely used technique. ${ }^{24}$ MRS studies have reported raised water content and choline-containing compounds (tCho) in malignant breast lesions which were shown to discriminate them from benign lesions and enhance the diagnostic specificity of MRI. ${ }^{25,26}$ Although, there has been considerable progress and promising results emerged from breast MRS in the last decade, further research should need to focus on advanced acquisition methods like use of parallel imaging, methods for development of coils which provide better comfort for patients and easy quantitative methods for the estimation of the tCho concentration before its inclusion in a clinical setting.

\subsection{Positron Emission Tomography (PET) Conjunction with Computer-Aided Tomography (PET-CT)}

PET is a molecular imaging modality in which compounds labelled with positron emitting radioisotopes are used to measure biological processes with only trace amounts of the radiolabeled probes. PET imaging is an important clinical modality for oncological, cardiovascular, and neurological applications. Over the years many types of PET radiotracers have been developed to noninvasively examine in vivo tumor metabolism, A frequently used PET radiotracer is $18 \mathrm{~F}$ fluorodeoxyglucose (FDG), approved by US Food and Drug Administration (FDA). FDG is transported into the cell via glucose transporters, but unlike glucose, FDG is not metabolized instead it is irreversibly phosphorylated by hexokinase and trapped within the cell. ${ }^{27}$ Because glucose transport is up regulated in most cancers in a phenomenon termed the Warburg effect ${ }^{28}$, FDG PET exploits 
greater uptake of FDG within most cancer cells versus normal tissue in order to visualize tumors. The uptake of FDG in breast cancer includes not only tumor cell density but also mitotic activity index, histologic grade, as well as other markers of aggressiveness. ${ }^{29}$ While FDG uptake positively correlates with pathologic complete response to neoadjuvant chemotherapy for patients with breast cancer, it also correlates inversely with prognosis. ${ }^{30}$ It usually takes between 30 and 60 minutes for the FDG to distribute throughout the body. Because highly active cancer cells absorb more glucose than normal cells, they appear brighter on PET scans. So, areas where a large amount of FDG accumulates, called 'hot spots' because they appear more intense than surrounding tissue, indicate that a high level of chemical activity or metabolism is occurring there. Areas of low metabolic activity appear less intense and are sometimes referred to as 'cold spots'. ${ }^{31}$ PET combined with CT known as PET-CT is the combination of two imaging techniques - called co-registration, fusion imaging or hybrid imagingallows information from two different types of scans to be viewed in a single set of images, producing highly detailed views of the body. PET-CT study is able to provide detail on both the anatomy and function of organs and tissues. This is accomplished by superimposing the precise location of abnormal metabolic activity (from PET) against the detailed anatomic image (from CT). Compared to conventional imaging techniques FDG PET-CT results in improved sensitivity for the detection of both lymph node infiltration and distant metastases in patients with breast cancer. ${ }^{32}$ The development of PET scanners dedicated to breast (Positron Emission Mammography) has improved spatial resolution and sensitivity, allowing its clinical application in the study of the primary tumor. ${ }^{33}$ PET holds a decisive role in the detection of distant metastases, tumor recurrence and evaluation of therapeutic response, regarding the diagnosis of primary breast lesions and locoregional staging it has a limited diagnostic value as compared with other imaging methods. ${ }^{34}$ The FDG-PET systems spatial resolution ranges between 5 and $8 \mathrm{~mm}$ therefore lesions smaller in size may not be fully resolved, and tracer concentrations will be underestimated due to partial volume effects. ${ }^{35}$ Rosi et. al. mentioned in the review article that tumors smaller than $10 \mathrm{~mm}$, the sensitivity of 18 F-FDG PET-CT is $25 \%$, while for tumors between $10 \mathrm{~mm}$ and $20 \mathrm{~mm}$ the sensitivity is $84 \% .{ }^{36}$ On the other hand, many studies show that hybrid imaging with 18F-FDG PET-CT provides information about the intake and glucose metabolism, which are increased in malignant lesions. ${ }^{37}$ In the era of individualized medicine $18 \mathrm{~F}$ FDG as a general indicator of glucose consumption remains the cornerstone of PET imaging in breast cancer, while several other radiopharmaceuticals are constantly being developed, serving as diagnostic and prognostic tools in the research of breast tumors. ${ }^{38}$ Back in 1998, 3-deoxy-3-[18F] fluorothymidine (18FLT) biomarker was developed and tested for in vivo imaging of cell proliferation, demonstrating that it was resistant to degradation in vivo, retained in proliferating tissues by the action of thymidine kinase 1 and produced high-contrast images of normal marrow and tumors. ${ }^{39}$ Even though in the clinical practice of breast cancer 18FLT is not a routine tool, it may play an important role in staging, monitoring, and prediction of response to therapy agents. ${ }^{40} \mathrm{~A}$ study demonstrated that 18FLT can detect changes in breast cancer proliferation as early as 1 week after 5fluorouracil, epirubicin, and cyclophosphamide therapy. ${ }^{41}$ Pio et al. compared 18F-FDG and 18F-FLT imaging in 14 patients with newly diagnosed primary or metastatic breast cancer for monitoring and predicting tumor response to chemotherapy. ${ }^{42}$ The group concluded that $18 \mathrm{~F}$-FLT may be more accurate than 18F-FDG two weeks after the end of the first course of chemotherapy for predicting longer-term efficacy of chemotherapy for women with breast cancer. PET with $16 \alpha-[18 \mathrm{~F}]$-fluoro-17 $\beta-$ estradiol (18F-FES) enables non-invasive visualization and quantification of estrogen receptors (ER) expression in all tumor lesions within a patient. In addition, this imaging technique can potentially provide in-vivo information about ER binding of endocrine drugs. ${ }^{43}$ In addition, a number of progesterone receptor PR-targeting radiotracers have been also investigated in preclinical and clinical studies. The most successful PR-targeted radiotracer is the progesterone analog 18F-fluoro-16, $17 \alpha-$ [(R)-(1'- $\alpha$-fury methylidene) dioxy]-19-norpregn-4-ene-3,20-dione (18F-FFNP). ${ }^{44}$ The first-in-human study designed to evaluate the feasibility of imaging tumor PRs by PET in breast cancer as well, the safety and dosimetry of $18 \mathrm{~F}$-FFNP. ${ }^{45}$ Some studies demonstrated that serial FFNP-PET imaging was more effective than FDG-PET and FES-PET in predicting response to estrogen deprivation therapy in preclinical models of $E R \alpha+$ breast cancer, introducing its role in a neoadjuvant setting so that a more aggressive treatment course can be implemented after surgery if the primary tumor is predicted to be endocrine-nonresponsive. ${ }^{46}$ Radiolabeled immunoglobulins (trastuzumab and pertuzumab), immunoglobulin fragments, $\mathrm{F}\left(\mathrm{ab}^{\prime}\right) 2$, diabodies, nanobodies and non-immunoglobulin scaffolds, affibody and designed ankyrin-repeat proteins devise a reliable and quantitative method for detecting HER2-positive cancer using PET ${ }^{47}$ Trastuzumab is a monoclonal antibody that targets HER2, which 
is used in HER2-positive breast cancer therapy. 89Zr-DFO-trastuzumab, 64Cu-DOTA-trastuzumab are an attractive option for antibody-based imaging agents due to their compatible half-life and they proved to be promising in detecting not only primary tumor, but also metastatic disease that conventional PET was unable to accomplish. ${ }^{48}$ PET helps physicians and their patients: gain a clear understanding of where and how aggressive the disease is, select a course of treatment, determine the effectiveness of treatments after just one cycle of treatment, eliminate unnecessary surgeries after treatment by distinguishing active tumors from residual masses. ${ }^{49}$ Research advances in molecular oncology continue to generate new targeted therapies contributing to the achievement of precision medicine. Molecular imaging probes and radiopharmaceuticals are important tools that can be used to visualize, characterize, quantify and monitor breast cancer treatment response. Continued clinical validation of these agents is necessary to fully achieve translation from initial preclinical status to FDA approval, reimbursement, and widespread clinical implementation with the ultimate goal of improving outcomes for breast cancer patients.

\section{CONCLUSION}

Breast cancer has become a major healthcare burden: screening programs are costly and difficult to organize, involving major logistic and quality control. Early diagnosis is key to the successful treatment of breast cancer and helping increase patient survival rate. With the expansion of tumor information provided by advanced examination techniques like mammography, ultrasound, MRI, and nuclear medicine imaging can further develop via greater individualization and precision. Although each of these techniques has their advantages and disadvantages, there are ways to improve these methods. Simultaneous, combined use of these imaging techniques for the early detection of breast cancer can be improved. This review provided a summary of the evidence regarding the current and future use of imaging to support patient decision making and decision made by surgical, radiation, and medical oncology experts. Along with existing evidence on how to appropriately use available techniques of precision imaging, a number of ongoing and future trials will refine the role of imaging in breast cancer to optimize the management and quality of life in women diagnosed with breast cancer.

\section{ACKNOWLEDGEMENT}

I would like to acknowledge my chemistry teacher, Sarah Barton and Alyson Levine, and my mother for their continued guidance and support during my literature search.

\section{REFERENCES}

[1] 11 Factors about breast cancer. Available from: https://www.dosomething.org/facts/11-facts-about-breastcancer.

[2] Munnink, TH Oude, et al. "Molecular imaging of breast cancer. The Breast. 2009; S66-S73.

[3] Goldsmith M, Koutcher JA, Damadian R. NMR in cancer, XIII: application of the NMR malignancy index to human mammary tumours. British Journal of Cancer 1978; 38(4): 547-54. Available from: https://doi.org/10.1038/bjc.1978.243.

[4] Lohrke J, Frenzel T, Endrikat J, et al. 25 Years of Contrast-Enhanced MRI: Developments, Current Challenges and Future Perspectives. Advances in Therapy 2016; 33(1): 1-28. Available from: https://doi.org/10.1007/s12325-015-0275-4.

[5] Myers KS, Kamel IR, Macura KJ. MRI-guided breast biopsy: outcomes and effect on patient management. Clinical Breast Cancer 2015; 15(2): 143-52. Available from: https://doi.org/10.1016/j.clbc.2014.11.003.

[6] Imschweiler T, Haueisen H, Kampmann G. et al. MRI-guided vacuum-assisted breast biopsy: comparison with stereotactically guided and ultrasound-guided techniques. European Radiology 2014; 24: 128-35. Available from: https://doi.org/10.1007/s00330-013-2989-5.

[7] Meeuwis C, Veltman J, van Hall HN, et al. MR-guided breast biopsy at 3T: diagnostic yield of large core needle biopsy compared with vacuum-assisted biopsy. European Radiology 2012; 22: 341-49. Available from: https://doi.org/10.1007/s00330-011-2272-6.

[8] Tourasse C, Khasanova E, Sebag P, Beregi JP. Ultrasound-guided vacuum-assisted breast biopsy with a small-caliber device: A multicenter consecutive study of 162 biopsied lesions. Tumori Journal 2019; 105(4): 312-18. Available from: https://doi.org/10.1177/0300891618784786. 
[9] Bleicher RJ, Ciocca RM, Egleston BL, et al. Association of routine pretreatment magnetic resonance imaging with time to surgery, mastectomy rate, and margin status. Journal of the American College of Surgeons 2009; 209(2):180-87. Available from: https://doi.org/10.1016/j.jamcollsurg.2009.04.010.

[10] Rajkumar JS., Dharmendra KR, Rajkumar A, et al. MRI before fistula surgery: will it change the operation? - a prospective study. Indian Journal of Surgery 2019; 81(2): 178-81. Available from: https://doi.org/10.1007/s12262-018-1857-6.

[11] Choi EJ, Choi H, Choi SA, Youk JH. Dynamic contrast-enhanced breast magnetic resonance imaging for the prediction of early and late recurrences in breast cancer. Medicine 2016; 95(48): e5330. Available from: https://dx.doi.org/10.1097\%2FMD.0000000000005330.

[12] Chitalia RD, Rowland J, McDonald ES, et al. Imaging Phenotypes of Breast Cancer Heterogeneity in Preoperative Breast Dynamic Contrast Enhanced Magnetic Resonance Imaging (DCE-MRI) Scans Predict 10-Year Recurrence. Clinical Cancer Research 2020; 26(4): 862-69. Avialable from: https://10.1158/10780432.CCR-18-4067.

[13] Gillman J, Toth HK, Moy L. The role of dynamic contrast-enhanced screening breast MRI in populations at increased risk for breast cancer. Women's Health 2014; 10(6): 609-22. Available from: https://doi.org/10.2217\%2FWHE.14.61.

[14] Schnall MD, Blume J, DeAngelis GA. et al. Diagnostic architectural and dynamic features at breast MR imaging: multicenter study. Radiology 2006; 238: 42-53. Available from: https://doi.org/10.1148/radiol.2381042117.

[15] Pepin KM, Ehman RL, McGee KP. Magnetic resonance elastography (MRE) in cancer: technique, analysis, and applications. Progress in Nuclear Magnetic Resonance Spectroscopy 2015; 90: 32-48. https://doi.org/10.1016/j.pnmrs.2015.06.001.

[16] Low G, Kruse SA, Lomas DJ. General review of magnetic resonance elastography. World Journal of Radiology 2016; 8(1): 59. Available from: https://dx.doi.org/10.4329\%2Fwjr.v8.i1.59.

[17] Patel BK, Samreen N, Zhou Y, et al. MR Elastography of the Breast: Evolution of Technique, Case Examples, and Future Directions. Clinical breast cancer 2020; Available from: https://doi.org/10.1016/j.clbc.2020.08.005.

[18] Hirano M, Satake H, Ishigaki S, Ikeda M, Kawai H, Naganawa S. Diffusion-weighted imaging of breast masses: comparison of diagnostic performance using various apparent diffusion coefficient parameters. American Journal of Roentgenology 2012; 198: 717-22. Available from: https://10.2214/AJR.11.7093.

[19] Durur-Subasi I. DW-MRI of the breast: a pictorial review. Insights Imaging 2019; 10: 61. Available from: https://doi.org/10.1186/s13244-019-0745-3.

[20] Partridge SC, Nissan N, Rahbar H, Kitsch AE, Sigmund EE. Diffusion-weighted breast MRI: Clinical applications and emerging techniques. Journal of Magnetic Resonance Imaging 2017; 45(2): 337-55. Available from: https://doi.org/10.1002/jmri.25479.

[21] Pereira NP, Curi C, Osório CA, et al. Diffusion-Weighted Magnetic Resonance Imaging of Patients with Breast Cancer Following Neoadjuvant Chemotherapy Provides Early Prediction of Pathological ResponseA Prospective Study. Scientific reports 2019; 9(1), 1-8. Available from: https://doi.org/10.1038/s41598019-52785-3.

[22] Baltzer P, Mann RM, Iima M. et al. Diffusion-weighted imaging of the breast-a consensus and mission statement from the EUSOBI International Breast Diffusion-Weighted Imaging working group. European Radiology 2020; 30: 1436-50. Available from: https://doi.org/10.1007/s00330-019-06510-3.

[23] Dager SR, Oskin NM, Richards TL, Posse S. Research applications of magnetic resonance spectroscopy (MRS) to investigate psychiatric disorders. Topics in magnetic resonance imaging: TMRI 2008; 19(2): 81. Available from: https://dx.doi.org/10.1097\%2FRMR.0b013e318181e0be.

[24] Stanwell P, Mountford C. In vivo proton MR spectroscopy of the breast. Radiographics 2007; 27(1), S253S266. Available from: https://doi.org/10.1148/rg.27si075519.

[25] Jagannathan NR. Application of in vivo MR methods in the study of breast cancer metabolism. NMR in Biomedicine 2019; 32(10): e4032. Available from: https://doi.org/10.1002/nbm.4032.

[26] Fardanesh R, Marino MA, Avendano D, Leithner D, Pinker K, Thakur SB. Proton MR spectroscopy in the breast: Technical innovations and clinical applications. Journal of Magnetic Resonance Imaging 2019; 50(4): 1033-46. Available from: https://doi.org/10.1002/jmri.26700. 
[27] Kawada K, Iwamoto M, Sakai Y. Mechanisms underlying 18F-fluorodeoxyglucose accumulation in colorectal cancer. World journal of radiology 2016; 8(11): 880-86. Available from: https://dx.doi.org/10.4329\%2Fwjr.v8.i11.880.

[28] Liberti MV, Locasale JW. The Warburg effect: how does it benefit cancer cells? Trends in biochemical sciences 2016; 41(3): 211-18. Available from: https://doi.org/10.1016/j.tibs.2015.12.001.

[29] Miyake KK, Nakamoto Y, Kanao S, et al. Journal Club: diagnostic value of (18)F-FDG PET/CT and MRI in predicting the clinicopathologic subtypes of invasive breast cancer. American Journal of Roentgenology 2014;203(2):272-79. Available from: https://10.2214/AJR.13.11971.

[30] Flavell RR, Naeger DM, Mari Aparici C, Hawkins RA, Pampaloni MH, Behr SC. Malignancies with low fluorodeoxyglucose uptake at PET/CT: pitfalls and prognostic importance: resident and fellow education feature. Radiographics 2016; 36(1): 293-94. Available from: https://doi.org/10.1148/rg.2016150073.

[31] Farwell MD, Pryma DA, Mankoff DA. PET/CT imaging in cancer: current applications and future directions. Cancer 2014; 20(22): 3433-45. Available from: https://doi.org/10.1002/cncr.28860.

[32] Aljabery F, Lindblom G, Skoog S, Shabo I, Olsson H, Rosell J, Jahnson S. PET/CT versus conventional CT for detection of lymph node metastases in patients with locally advanced bladder cancer. BMC urology 2015; 15(1): 1-6. Available from: https://doi.org/10.1186/s12894-015-0080.

[33] Narayanan D, Berg WA. Dedicated breast gamma camera imaging and breast PET: current status and future directions. PET Clinics 2018; 13(3), 363-81.Available from: https://doi.org/10.1016/j.cpet.2018.02.008.

[34] Iakovou I, Giannoula E, Gkantaifi, A, Levva, S, Frangos S. Positron emission tomography in breast cancer: 18 F-FDG and other radiopharmaceuticals. European Journal of Hybrid Imaging 2018; 2(1): 20. Available from: https://doi.org/10.1186/s41824-018-0039-x.

[35] Lybum ID, Brown EF. Application of positron emission tomography - computerized tomography in breast cancer. In: Michell JM (ed) Contemporary Issues in cancer imaging. Breast Cancer 2010. Cambridge University Press, New York, pp 218-24.

[36] Avril N, Rosi CA, Schelling M, et al. Breast imaging with positron emission tomography and fluorine-18 fluorodeoxyglucose: use and limitations. Journal of Clinical Oncology 2000; 18: 3495-502. Available at: http://citeseerx.ist.psu.edu/viewdoc/download?doi=10.1.1.1033.9626\&rep=rep1\&type=pdf.

[37] Giammarile F, Castellucci P, Dierckx R, et al. Non-FDG PET/CT in Diagnostic Oncology: a pictorial review. European Journal of Hybrid Imaging 2019; 3(1): 20. Available from: https://doi.org/10.1186/s41824-019-0066-2.

[38] Signore A, Lauri C, Auletta S, et al. Radiopharmaceuticals for Breast Cancer and Neuroendocrine Tumors: Two Examples of How Tissue Characterization May Influence the Choice of Therapy. Cancers (Basel). 2020; 12(4): 781. Available from: https://doi.org/10.3390/cancers 12040781.

[39] Shields AF, Grierson JR, Dohmen BM, et al. Imaging proliferation in vivo with [F-18] FLT and positron emission tomography. Nature medicine 1998; 4(11): 1334-36. Available from: https://doi.org/10.1038/3337.

[40] Cervino AR, Burei M, Mansi L, Evangelista L. Molecular pathways and molecular imaging in breast cancer: an update. Nuclear Medicine and Biology 2013; 40(5): 581-91. Available from: https://doi.org/10.1016/j.nucmedbio.2013.03.002.

[41] Kenny L, Coombes RC, Vigushin DM, Al-Nahhas A, Shousha S, Aboagye EO. Imaging early changes in proliferation at 1-week post chemotherapy: a pilot study in breast cancer patients with 3'-deoxy-3'- [18 F] fluorothymidine positron emission tomography. European journal of nuclear medicine and molecular imaging 2007; 34(9): 1339-47. Available from: https://doi.org/10.1007/s00259-007-0379-4.

[42] Pio BS, Park CK, Pietras R, et al. Usefulness of 3'-[F-18] fluoro-3'-deoxythymidine with positron emission tomography in predicting breast cancer response to therapy. Molecular Imaging and Biology 2006; 8(1): 36-42. Available from: https://doi.org/10.1007/s11307-005-0029-9.

[43] van Kruchten M, de Vries EG, Brown M, et al. PET imaging of oestrogen receptors in patients with breast cancer. The Lancet Oncology 2013; 14(11): e465-e475. Available from: https://doi.org/10.1016/S14702045(13)70292-4.

[44] Dalm SU, Verzijlbergen JF, De Jong M. Receptor targeted nuclear imaging of breast cancer. International Journal of Molecular Sciences 2017; 18(2): 260. Available from: https://doi.org/10.3390/ijms18020260. 
[45] Dehdashti F, Laforest R, Gao F, et al. Assessment of progesterone receptors in breast carcinoma by PET with 21-18F-fluoro-16 $\alpha, 17 \alpha$-[(R)-(1'- $\alpha$-furylmethylidene) dioxy]-19-norpregn-4-ene-3,20-dione. Journal of Nuclear Medicine 2012; 53(3): 363-70. Available from: https://doi.org/10.2967/jnumed.111.098319.

[46] Chan SR, Fowler AM, Allen JA et al. Longitudinal noninvasive imaging of progesterone receptor as a predictive biomarker of tumor responsiveness to estrogen deprivation therapy. Clinical Cancer Research 2015; 21(5): 1063-70. Available from: https://DOI:10.1158/1078-0432.CCR-14-1715.

[47] Goldstein R, Sosabowski J, Vigor K, Chester K, Meyer T. Developments in single photon emission computed tomography and PET-based HER2 molecular imaging for breast cancer. Expert review of anticancer therapy 2013; 13(3): 359-73. Available from: https://doi.org/10.1586/era.13.11.

[48] Henry KE, Ulaner GA, Lewis JS. Human epidermal growth factor receptor 2-targeted PET/single-photon emission computed tomography imaging of breast cancer: noninvasive measurement of a biomarker integral to tumor treatment and prognosis. PET clinics 2017; 12(3): 269-88. Available from: https://doi.org/10.1016/j.cpet.2017.02.001.

[49] Jakub JW, Pendas S, Reintgen DS. Current status of sentinel lymph node mapping and biopsy: facts and controversies. The Oncologist 2003; 8(1): 59-68. Available from: https://doi:0.1634/theoncologist.8-1-59.

\section{AUTHORS' BIOGRAPHY}

Arya Bhushan is currently a senior at Ladue Horton Watkins High School in St. Louis, Missouri. In his free time, he enjoys playing chess, basketball, and tennis.

Citation: Arya Bhushan, Imaging Technologies - A Tool for Early Breast Cancer Diagnostic. International Journal of Research Studies in Biosciences. 2021; 9(2): 22-29. DOI: https://doi.org/10.20431/2349. 0365.0902004.

Copyright: (C) 2021 Authors. This is an open-access article distributed under the terms of the Creative Commons Attribution License, which permits unrestricted use, distribution, and reproduction in any medium, provided the original author and source are credited. 